\title{
Erratum: Natural hyperbolicity in layered hexagonal crystal structures [Phys. Rev. B 103, 035425 (2021)]
}

\author{
Ali Ebrahimian and Reza Asgari \\ (Received 1 February 2022; published 14 February 2022)
}

DOI: 10.1103/PhysRevB.105.079901

Due to the unknown lifetime broadening value, we demonstrated only the interband contribution in Fig. 6. By mistake, we forgot to mention that point regarding this particular result of our paper. We comprehensively investigated the intraband transition contribution of the dielectric tensor of $\mathrm{Li}_{2} \mathrm{KN}$. First of all, $\mathrm{Li}_{2} \mathrm{KN}$ is a semimetal with tiny Fermi surfaces at $\Gamma$ and $K$ points and a small density of states. Therefore, the intraband contribution has a negligible effect at mid and high energy of the dielectric tensor and does not change the hyperbolic regions even at low frequency, which are discussed in our paper regarding Fig. 6. Therefore, the caption for Fig. 6 might be amended as follows:

FIG. 6. (a) The calculated bulk electronic band structure and (c) the interband contribution of the energy loss function to the in-plane ( $x$-polarized) and out-of-plane (z-polarized) incident light for $\mathrm{Li}_{2} \mathrm{KN}$. (b) The imaginary and (d) real parts of the interband dielectric tensor to the in-plane ( $x$-polarized) and out-of-plane ( $z$-polarized) incident light for $\mathrm{Li}_{2} \mathrm{KN}$. The spectral regions for type-I and type-II hyperbolicity are marked with a green and yellow background, respectively. The type-I hyperbolic frequency regime is broader than the type-II hyperbolic window with a hyperbolicity onset in the visible region at $2.3 \mathrm{eV}$. The transition from type I to type II occurs at $2.79 \mathrm{eV}$, where $\operatorname{Re} \varepsilon_{x x}(\omega)$ and $\operatorname{Re} \varepsilon_{z z}(\omega)$ vanish with an opposite sign slope. Furthermore, the loss function components show small values, meaning that there is no energy loss when the material is hyperbolic. Notice that owing to the unknown lifetime broadening of the plasmon mode, the intraband contribution is not shown here; although, our accurate numerical results show that the hyperbolic regions remain intact by including the lifetime broadening up to $0.1 \mathrm{eV}$. 